\title{
Analysis on the Characteristics of Flower Traditional Chinese Medicine for Preventing and Treating Cerebral Ischemia
}

\author{
Li-ling XIANG ${ }^{a}$, Ming-san MIAO ${ }^{b, *}$, Ya-juan WEN and Jing-yi QIAO \\ Department of Pharmacology, Henan University of Chinese Medicine, Zhengzhou, \\ 450046, China. \\ axiangliling168@163.com \\ bmiaomingsan@163.com \\ "Corresponding author
} Keywords: Flower Traditional Chinese Medicine, Cerebral Ischemia, Effective
Components, Flavonoids.

\begin{abstract}
Objectives: To investigate the characteristics and mechanism of flower traditional Chinese medicine for preventing and treating cerebral ischemia. methods: To summarize active components of flower traditional Chinese medicine on cerebral ischemia model and the characteristics of prevention and treatment of cerebral ischemia, summing up the mechanism of flower traditional Chinese medicine on cerebral ischemia. results: The effective components of flower traditional Chinese medicine for prevention and treatment of cerebral ischemia are mainly flavonoids, then Phenolic acids and polysaccharides, Its efficacy is attributed to blood stasis, heat clearing and detoxifying, all kinds of flowers are common in the prevention and treatment of cerebral ischemia. conclusions: The effects of flower traditional Chinese medicine on the prevention and treatment of cerebral ischemia are multiple pathways, multi targets and multi links.
\end{abstract}

\section{Introduction}

Cerebrovascular disease is a common and frequently occurring disease with high mortality and morbidity, It is the world's three leading cause death with heart disease and cancer, which had a serious threat to human life and health. According to the different causes of the disease, it can be divided into ischemic and hemorrhagic, and, the former incidence of a disease is far higher than the latter. The underlying mechanism and the ultimate cause was ischemia and hypoxia of ischemic cerebrovascular disease, cerebral ischemia reperfusion can restore nerve function, but after the recovery of blood supply, the excessive free radicals attack to the tissue, which can cause cell damage from ischemia reperfusion injury, and induced the serious threat of patients [1]. Clinical use antiplatelet aggregation, expansion, anticoagulation, vascular expansion of the treatment, These treatments have the advantages of quick response and strong pertinence, but the disadvantage is that most of them will have side effects and drug resistance, and can not reach the therapeutic effect of multiple targets. Traditional Chinese medicine has the characteristics of multi way, multi targets and multi links, Through a variety of mechanisms to interfere with the pathological process of cerebral ischemia injury, Therefore, it has a protective effect on brain tissue and has fewer side effects, so it is paid more and more attention. Fragrance of flower Chinese herbal medicine can be adjusted people feelings, The fragrant smelling has multiple effects, it can pass the meridians, stimulate the circulation of the blood and cause the muscles and 
joints to relax, It has a wide range of resources and potential value of development and utilization, In recent years, the flower traditional Chinese medicine on the prevention and treatment of cerebral ischemia showed a good effect, the related research is summarized as follows:

\section{Effective Components of Flower Chinese Herbal Medicine on cerebral Ischemia}

\section{Flavonoids}

Flavonoids are secondary metabolites produced by natural selection in plants, and widely exists in nature, Flavonoids are effective components of many Chinese herbal medicines, which have a wide range of biological activities, it can effectively remove oxygen free radicals in the body, improve blood circulation, lowering cholesterol, reduce blood sugar and anti-oxidation. It was widely used in clinical treatment of cardiovascular and cerebrovascular diseases [2]. In recent years, through the study on the pharmacological effects of flower Chinese herbal medicine, it was found that flavonoids can prevent and cure cerebral ischemia and its complications. The mechanism may be related to its anti free radical or antioxidant.

Total Flavonoids of Chinese Trumpet Creeper. In the pharmacological research of Campsis grandiflora, it was found to improve blood circulation, relaxing coronary artery, inhibiting thrombosis, antioxidant and anti-inflammatory effects [3], Fang Xiaoyan observed the protective effect of chinese trumpet creeper flavonoids on cerebral ischemia reperfusion injury in rats, The study found that chinese trumpet creeper flavonoids can significantly improve the symptoms of nerve defect in rats, reduced neurological deficit score, And significantly reduce the degree of pathological damage of brain tissue, It has protective effect on transient cerebral ischemia reperfusion in rats[4], Fang further observated the effect of chinese trumpet creeper flavonoids on inflammatory factors and chemokines in rat brain tissue and NF- $\kappa \mathrm{B} / \mathrm{iNOS}-\mathrm{COX}-2$ pathway in cerebral ischemia rats model, Found chinese trumpet creeper flavonoids inhibited inflammatory factors and chemokine levels in brain tissue of rats , significantly decreased iNOS activity and NO level in brain homogenate, significantly decreased the number of NF- $\kappa B p 65$ and COX-2 positive cells. Summarize chinese trumpet creeper flavonoids inhibit the inflammatory reaction of cerebral ischemia reperfusion injury, The mechanism was mainly to inhibit the activation of $\mathrm{NF}-\kappa \mathrm{B} / \mathrm{iNOS}-\mathrm{COX}-2$ signaling pathway [5-6].

Breviscapine. The effective components of Erigeron breviscapus are flavonoids, mainly breviscapine, Flavonoids in Erigeron breviscapus can improve blood circulation, antioxidation, anti platelet aggregation and blood viscosity[7], The effects of scutellarin on the expression of HSP70 in ischemic brain tissue of rats with focal cerebral ischemia reperfusion injury was observed by [8], The results showed that scutellarin could significantly enhance the expression of HSP70 protein and mRNA in brain tissue of rats with ischemia-reperfusion injury. The mechanism of Breviscapine Injection on cytochrome CYP4A in rats with cerebral ischemia reperfusion injury [9], The results showed that Breviscapine injection can reduce the mortality of $168 \mathrm{~h}$ in rats with brain injury and reduce the infarct volume, inhibited the expression of CYP4A3, CYP4A8 and reduceed the content of eNOS in brain tissue, significantly improved cerebral ischemia reperfusion injury in rats.

Carthamus Tinctorius Flavonoids. By studying the mechanism of the active ingredient of Carthamus tinctorius against cerebral ischemia reperfusion injury[10], 
The results showed that the compounds of the quinone type in Carthamus tinctorius had strong activity against cerebral ischemia, Mainly through the anti platelet aggregation, Improved vascular occlusion, anti free radical damage and anti excitatory amino acid toxicity. Objective to study the effects of Carthamus tinctorius injection on the expression of Bcl-2 protein, Bax and Bcl-XL in cerebral cortex of rats with cerebral ischemia reperfusion injury[11], The results showed that Carthamus tinctorius injection could significantly inhibit the expression of Bax positive cells and promote the expression of Bcl-2, Bcl-XL positive cells in the ischemic area of brain tissue, The results showed that Carthamus tinctorius injection could increase the expression of $\mathrm{Bcl}-2$, Bcl-XL protein and inhibit the expression of Bax protein to reduce the apoptosis of brain cells and protect the brain tissue of rats with ischemia-reperfusion injury.

Camellia Flavonoids. Protective effects of Camellia flavonoids on ischemic brain injury, It was found that the Camellia flavonoids could prolong the duration of the decapitation and the survival time under hypoxia[12], improveing learning and memory dysfunction after ischemia, reduce the content of MDA and NO in brain tissue after ischemia, Inhibited LDH activity, And can reduce the brain water content and vascular permeability, improved the pathological changes of brain tissue, The mechanism of anti cerebral ischemia may be related to anti free radical and inhibiting the formation of NO.

\section{Other Compounds}

Vinca Alkaloids. Effects of catharanthus roseus extraction on Hemorheology and antioxidant capacity in rats with cerebral ischemia[13], It was found that the catharanthus roseus of vincamine could improve the hemorheology of rats with cerebral ischemia, enhanced erythrocyte deformability, inhibited platelet aggregation, reduce blood viscosity and prevented thrombosis, At the same time, it could improve SOD activity and free radical scavenging.

Chlorogenic Acid. Study on the intervention effect of chlorogenic acid from honeysuckle extraction on cerebral ischemia reperfusion model in mice and rats, It was found that chlorogenic acid can dump free radicals, inhibit inflammatory reaction after cerebral ischemia, and improved a series of mediators and cytokines induced by inflammation, Scavenging oxygen free radicals, Protected tissue cells from oxidative stress, And can inhibit the apoptosis of nerve cells, promote angiogenesis, Protected ischemic penumbra, effectively improve cerebral ischemic injury.

Carthamus Tinctorious Polysaccharide. To study the protective effect and possible mechanism of Carthamus tinctorious polysaccharide on cerebral ischemia reperfusion injury in rats, The focal cerebral ischemia reperfusion model was established by suture method in rats, Carthamus tinctorious polysaccharide can improve neurological deficits in rats, reduced cerebral infarction volume and brain water content, reduced the content of TNF- $\alpha$ and IL- $1 \beta$ in brain tissue, and increased the cotent of IL-10, significantly inhibited the expression of Caspase-3 and PARP.

\section{Effects of Flower Chinese Herbal Medicine on Prevention and Treatment of Cerebral Ischemia}

It can be seen from the above that the effective components of traditional Chinese medicine for treating cerebral ischemia are mainly concentrated in flavonoids, It also includes some phenolic acids, polysaccharides and other ingredients. The efficacy focused on promoting blood circulation and removing blood stasis, heat clearing and detoxicating, Traditional Chinese medicine treatment mainly were blood stasis, heat 
clearing and detoxifying method, In according with the etiology and pathogenesis of stroke. Chinese medicine believes that the occurrence of ischemic stroke mainly with wind, phlegm, blood stasis, Qi deficiency, and so on. The blood stasis, heat toxin is an important factor [14]. With the deepening of the understanding of traditional toxin, it is suggested that the treatment of stroke by poison, so as to improve the curative effect of stroke, this has become a hot topic in the etiology and treatment of stroke. Traditional Chinese medicine has achieved good results by using the prescription of promoting blood circulation to remove blood stasis and clearing away heat and removing toxin, which has been widely recognized. In the experimental study, we may focus on the extraction of active ingredients from blood circulation and clearing away heat and removing toxin flowers in traditional Chinese medicine, the etiology and pathogenesis of the cerebral ischemia, by observing animal behavior, morphology, detection of inflammatory factors, biochemical indicators, to study the effect of cerebral ischemia [15]. Through the comprehensive analysis of the effective mechanism of Chinese herbal medicine on the prevention and treatment of cerebral ischemia, it can be seen that there are similarities between the effective components of flower traditional Chinese medicine in the prevention and treatment of cerebral ischemia, This helps to study on the molecular and genetic level of mechanism of flower traditional Chinese medicine effective components on cerebral ischemia, based on the overall effect of confirmation, selected high selectivity, low dose, high intensity of effects of Chinese medicine from the etiology and pathogenesis.

\section{Discussion}

Traditional Chinese medicine are often taken from a part of a plant or animal body, such as the use of plant roots, stem, leaves, flowers or fruits, etc. non medicinal parts are often discarded as waste, Flower traditional Chinese medicine is very useful, However, due to various reasons, some doctors and researchers gradually unfamiliar to it, mostly only applied to herbal tea series of pieces, and has not been fully utilized to play its original medicinal value. Modern pharmacological studies have found that Chinese herbal plants flower in the prevention and treatment of disease have a better therapeutic effects, we reviewed the relevant literature, summarized the effects of flower traditional Chinese medicine on cerebral ischemia, and determined the future research direction according to the research progress of flower traditional Chinese Medicine.

\section{Comprehensive Utilization of Flower Traditional Chinese Medicine Resources}

Through the comparative analysis of chemical analysis and pharmacological experiments and clinical observation of different parts of some plants, they can be expanded or applied to medicinal parts. Modern pharmacological studies have proved that the traditional Chinese medicine which has the function of resisting cerebral ischemia can be used for promoting blood circulation and removing blood stasis, clearing away heat and toxic substances, It can be inferred that the medicinal parts of this kind of traditional Chinese medicine are non flower parts such as root or rhizome, The correspond to the non medicinal parts of flowers have similar efficacy, which contains the active ingredients are basically the same, but there is a certain difference in content, it was found that the flowers have a wide range of pharmacological effects. However, the flowers are often discarded as waste materials, and their medicinal value was not fully utilized, It can be extracted and separated from a large number of flower resources, and its active ingredients as raw materials. Therefore, we can consider the 
further development of this kind of traditional Chinese medicine, such as flowers and stems and leaves, turning the waste into treasure, use resources rational.

\section{Developments of Chinese Herbal Medicine for Prevention and Treatment of Cerebral Ischemia}

Currently anti cerebral ischemia flower composition of preparation was less in the market, Such as erigeron injection, compound breviscapine dripping pills, Gu Hong injection, Danhong injection for the treatment of ischemic cardiovascular and cerebrovascular diseases. The products are few, the curative effect is not obvious, and it can be used to study the pharmacological effects of activating blood circulation and clearing away the active components in the parts of the traditional Chinese medicine, such as extracting promoting blood circulation and removing blood stasis and clearing away heat and toxic substances, To study the mechanism of its prevention and treatment of cerebral ischemia, and to develop its potential medicinal value, to develop a new anti ischemic drugs.

\section{Acknowledgement}

The research work is supported by The national "eleven five" supported program (2008BAI53B09); Henan Science and Technology Innovation Team (2012IRTSTHN011) ; science and technology innovation team of Zhengzhou city (131PCXTD612); The key Medical Laboratory for The transformation of Chinese Medicine of Zhengzhou City(121PYFZX1820).

\section{References}

[1] Xinxin Zhang, Xuanji Xue, Jing Zhao, et,al. Diosgenin attenuates the brain injury induced by transient cerebral ischemia-reperfusion in rats[J]. Steroids, 2016,113,103-112.

[2] Zhang Huaimin, Yang Hong, Zheng Haizhou, Research progress of natural flavonoids in prevention and treatment of cardiovascular and cerebrovascular diseases[J]. Chin J New Drugs Clin Rem, 2016,35 (10):704-708.

[3] Jiang Lingli, Miao Mingsan, Chemistry, Pharmacology and Clinical Application Characteristics of Lingxiaohua[J]. china journal of chinese medicine, 2014, 29(194):1016-1018.

[4] Fang Xiaoyan, Wu Suhui, et, al. Protective Effects of Campsis Flos Total Flavonoids on Cerebral Ischemia reperfusion Injury in rats $[\mathrm{J}]$. Chinese Journal of Experimental Traditional Medical Formulae, 2016, 22 (16):109-112.

[5] Fang Xiaoyan, Li Yucheng, et, al. Influence of total flavonoids of Campsis grandifloraon inflammation cytokines and chemokines in brain tissue of rats with cerebral ischemic $[\mathrm{J}]$. Chinese Journal of traditional chinese medicine, 2016, 09:3481-3483.

[6] Fang Xiaoyan, Zuo Ting, et, al. Effects of total flavone of Campsis grandifl ora K. Schum. on signal pathway NF- $\kappa \mathrm{B} / \mathrm{i}$ NOS-COX-2 in cerebral ischemia-reperfusion injury rats[J]. Chinese Journal of traditional chinese medicine, 2016, 08:3321-3324.

[7] Zhu Fayan, Yang Fang, et, al. Research progress of flavonoids in Erigeron breviscapus [J]. Anhui Agricultural Sciences, 2012, 10:5853-5857. 
[8] Jin Guilan, Tan Huilin, et, al. Protective effects of breviscapine on focal cerebral ischemia in mice and expression of P-glycoprotein in brain tissue [J]. Pharmacology and clinic of traditional Chinese medicine, 2015, 01:76-79.

[9] Cao Yu, Li Zhaofei, et, al. Effects of Breviscapine Injection on CYP4A in rats with cerebral ischemia reperfusion injury [J]. Chinese Journal of Experimental Traditional Medical Formulae, 2016, 03:1-7.

[10]Xia Ying, Zhao Lijian, Zhu Huiyuan, Study on the mechanism of active components of Carthamus tinctorius against cerebral ischemia reperfusion injury [J]. The world's latest medical information abstracts, 2016, 34:135-136.

[11] Chen Qian, Feng Fei, et, al. Effects of safflower injection on Bax and Bcl-XL/Bcl-2 in cerebral cortex of rats with cerebral ischemia reperfusion injury [J]. Chinese Journal of pharmacology, 2015, 01:15-18.

[12]Luo Shengyong, Fang Ming. Effects of total flavonoids of Camellia japonica on apoptosis of cerebral ischemia reperfusion injury in rats $[\mathrm{J}]$. Chinese Pharmaceutical Journal, 2010, 09:665-668.

[13] Jin Pingkui, Jiang Xiangming, Effects of Changchun flower extract on Hemorheology and antioxidant capacity in rats with cerebral ischemia [J].2012, 09:26-28.

[14]Cheng Xiao, Zhang Xiaoli, Miao Mingsan. Prevention and treatment of cerebral ischemia by promoting blood circulation and removing blood stasis and clearing away heat and toxic substances [J]. Chinese Journal of traditional Chinese medicine, 2012, 05:615-619.

[15]Li Ruiqi, Bai Ming, Miao Mingsan. Analysis of the characteristics of Chinese herbal medicine against cerebral ischemia $[\mathrm{J}]$. Chinese Journal of traditional Chinese medicine, 2013,08:1184-1187. 